\title{
The highest resolution near infrared spectrum of the imaged planetary mass companion $2 \mathrm{M} 1207 \mathrm{~b}^{\star, \star \star}$
}

\author{
J. Patience ${ }^{1}$, R. R. King ${ }^{1}$, R. J. De Rosa ${ }^{1}$, and C. Marois ${ }^{2}$ \\ 1 Astrophysics Group, School of Physics, University of Exeter, Stocker Road, Exeter EX4 4QL, UK \\ e-mail: [patience;rob; derosa]@astro.ex.ac.uk \\ 2 National Research Council Canada, Herzberg Institute of Astrophysics, BC V9E 2E7, Victoria, Canada \\ e-mail: christian.marois@nrc-cnrc.gc.ca
}

Received 27 January 2010 / Accepted 20 March 2010

ABSTRACT

\begin{abstract}
Direct-imaging searches for planets reveal wide orbit planets amenable to spectroscopy, and their atmospheres represent an important comparison to the irradiated atmospheres of Hot Jupiters. Using AO integral field spectroscopy of $2 \mathrm{M} 1207 \mathrm{~b}$, the shape of the continuum emission over the $J, H$, and $K$ bands from the atmosphere of this young, planetary mass companion is measured in order to compare with atmospheric and evolutionary models, and objects of similar temperature in young clusters and the field. The $2 \mathrm{M} 1207 \mathrm{~b}$ spectrum has the highest spectral resolution $(R \sim 300-1500)$ and largest wavelength coverage, including the first $J$-band spectrum, for this benchmark object. The high signal-to-noise of the data allow a clear identification of signatures of low surface gravity, and comparison with a grid of AMES-Dusty models reveals a best-fit effective temperature of $T_{\text {eff }}=1600 \mathrm{~K}$ with a preferred surface gravity of $\log g=4.5$. The $J$-band flux is depressed relative to nearly all L-type objects, and the detailed shape of the absorption features across the $H$-band exhibit differences from the model predictions. The possible origins of $2 \mathrm{M} 1207 \mathrm{~b}$ and its low luminosity are examined with the new data and analysis which suggest that extinction from a disk with large grains is a viable scenario and is preferred over scatttering off an optically thick disk. The $2 \mathrm{M} 1207 \mathrm{~b}$ spectrum presents an important comparison for the types of features which may be present in upcoming spectra of the atmospheres of planets imaged in orbit around stellar primaries.
\end{abstract}

Key words. planetary systems - brown dwarfs - planets and satellites: atmospheres - binaries: close -

techniques: high angular resolution

\section{Introduction}

Over 400 extrasolar planets are currently known ${ }^{1}$, with most systems detected indirectly in orbits closer than that of Jupiter. For indirectly detected planets, the rare combination of a favourable geometry resulting in a transit and a very bright host star for high signal-to-noise data is required to obtain a planetary spectrum either in transmission (Charbonneau et al. 2002) or emission (Charbonneau et al. 2005; Deming et al. 2005). Although only two transiting planets have been investigated spectroscopically, transmission studies have shown the possibility of methane absorption (Swain et al. 2008) or Rayleigh scattering (Sing et al. 2008) and a haze layer (Pont et al. 2008), and emission studies have observed the presence (Grillmair et al. 2008) and absence (Richardson et al. 2007) of water band absorption in different systems.

The atmospheres of directly-imaged planets represent a complementary case study to the transiting planet spectroscopy, uncontaminated by the physical effects of extreme proximity to the host star. In some cases, spectroscopy of directly imaged planets is feasible. The first confirmed directly-imaged planets orbiting

* Based on observations obtained at the Paranal Observatory, Chile for ESO program 078.C-0800(B).

$\star \star$ Spectra are only available in electronic form at the CDS via anonymous ftp to cdsarc.u-strasbg. fr $(130.79 .128 .5)$ or via http://cdsweb.u-strasbg.fr/cgi-bin/qcat?]/A+A/517/A76

1 www.exoplanet.eu normal stars (Kalas et al. 2008; Marois et al. 2008) and the first imaged planetary mass companion (Chauvin et al. 2004) present the opportunity for spectroscopic analysis of young planet atmospheres. For technical reasons, targets observed in imaging planet searches are typically younger than stars searched for planets indirectly, and this selection criterion enables an investigation of the early evolution of planetary atmospheres. The first spectrum of HR $8799 \mathrm{c}$ in the $L$-band suggests differences in the continuum shape from theoretical models (Janson et al. 2010), possibly due to non-equilibrium $\mathrm{CO} / \mathrm{CH}_{4}$ chemistry and indicating the importance of measuring the atmosphere over an even larger wavelength range. This paper presents the results of the highest resolution $J, H, K$ spectrum of $2 \mathrm{M} 1207 \mathrm{~b}$ which should provide a high signal-to-noise comparison spectrum to planets imaged in orbit around stellar primaries.

\section{Target}

The target for this study is one of the lowest mass companions imaged to date (Chauvin et al. 2004, 2005). 2M1207b is confirmed as a companion based on multi-epoch imaging (Song et al. 2006) and, considering the distance of $52.4 \pm 1.1 \mathrm{pc}$ (Ducourant et al. 2008), orbits the host brown dwarf at a projected separation of $46 \mathrm{AU}$, somewhat wider than the SunNeptune distance. The companion has apparent magnitudes of $J=20.0 \pm 0.2$ (Mohanty et al. 2007), $H=18.09 \pm 0.21$, and $K_{\mathrm{S}}=16.93 \pm 0.11$ (Chauvin et al. 2004). Combining the system 
Table 1. SINFONI observations.

\begin{tabular}{lcccccllll}
\hline \hline Source & Grating & Scale & NDIT & DIT & \#Exp. & Standard (HIP) & Standard $T_{\text {eff }}(\mathrm{K})$ & Dates & Project ID \\
\hline 2M1207A+b & $H+K$ & 100 & 1 & $300 \mathrm{~s}$ & 24 & 54930,62566 & 29000,15000 & 28 Jan. 07, 07 Feb. 07 & $078 . \mathrm{C}-0800(\mathrm{~B})$ \\
& $J$ & 100 & 1 & $600 \mathrm{~s}$ & 6 & 59951 & 16400 & 22 Feb. 07 & 078.C-0800(B) \\
\hline
\end{tabular}

age and theoretical cooling tracks (Chabrier et al. 2000; Baraffe et al. 2002, 2003) with the target photometry (Chauvin et al. 2005 ) indicates a mass of $\sim 5 M_{\text {Jup }}$, whereas with the spectroscopically estimated effective temperature, a mass of 6-10 $M_{\mathrm{Jup}}$ is predicted (Mohanty et al. 2007). The masses depend critically on the system age, estimated to be $8 \mathrm{Myr}$ for 2M1207 from its membership in the TW Hydra association (Gizis 2002); the unusually well-determined age of this low mass companion eliminates some degeneracies in model comparisons. Given the large difference in mass ratio of the 2M1207 system compared to the HR 8799 and Fomalhaut systems, their formation mechanisms may be different, with 2M1207 representing the tail of the binary distribution and the A-star planets examples that formed in a disk via core accretion (e.g., Pollack et al. 1996) or gravitational instability (e.g., Boss 1997). Despite the possibility of different origins, 2M1207 b is a benchmark object for the study of young planetary atmospheres.

\section{Observations}

The observations were taken with SINFONI, an AO-equipped integral field spectrograph mounted at the Cassegrain focus of the VLT (Bonnet et al. 2004; Eisenhauer et al. 2003; Thatte et al. 1998) in program 078.C-0800(B) (PI: Thatte). Details of the observations are given in Table 1 . The primary object was used for the AO correction. Of the four gratings available in SINFONI, the $J(R \sim 2000)$ and $H+K(R \sim 1500)$ were used, which yields higher spectral resolution than previous results (Chauvin et al. 2004; Mohanty et al. 2007) and the first $J$-band spectrum. The 100 mas spatial scale was employed as it is fine enough to separate the pair, but coarse enough to concentrate the flux of the faint companion. The field-of-view at this scale is $3{ }^{\prime \prime} 0 \times 33^{\prime \prime} 0$ and included both components.

The observing sequence included a set of exposures on the target at several offset positions on the array. Observations of an early type star followed each target sequence to correct for telluric features and the instrument response. Additional calibrations were obtained to measure the wavelength scale, the detector dark current, distortion, and quantum efficiency variations.

\section{Data analysis}

The data were reduced with the Gasgano (Gebbinck et al. 2007) implementation of the SINFONI data reduction pipeline (Modigliani et al. 2009; Dumas et al. 2007). With a set of detector calibration files, corrections for the dark current, linearity response, and bad pixels were measured and applied to all data files. Two effects of the instrument optics were also corrected the distortion of the pixel scale across the field and the non-linear dispersion of the gratings. After the detector and optics processing was completed, the array data were converted into data cubes consisting of a stack of images, each covering a slice of wavelength. Across the entire wavelength range, a stack of images were constructed, composed of 700-2000 wavelength slices, depending on the band. Binning was required for the $J$-band data.

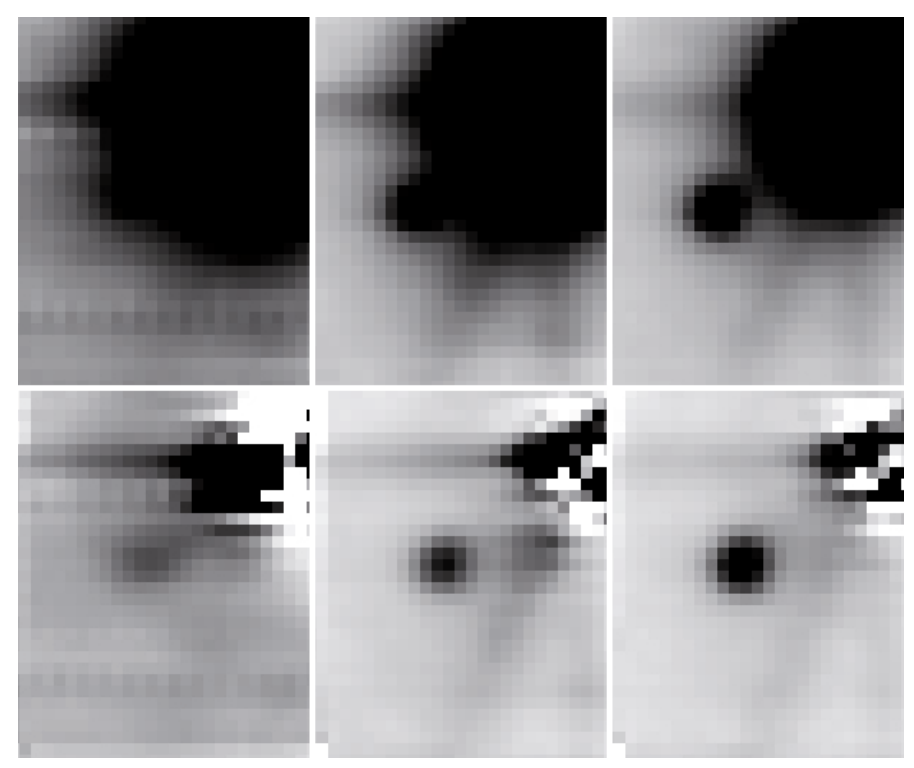

Fig. 1. The $J, H, K$ wavelength collapsed images before and after the alignment and subtraction process. For the $H$ - and $K$-band portions of the data, the companion is very distinct; although the $J$-band image is lower signal-to-noise, the companion is still clearly evident, enabling the spectrum to be measured in the binned data cube.

The final steps applied to the data cube involved sky subtraction, combining the offset positions, radial subtraction of the host object, and tracing the path of the companion spectrum through the data cube. The targets were observed at different positions on the array in an ABBA pattern sampling two areas on the array. The cubes with the target in one portion of the array were averaged and then used as the sky to subtract off the individual cubes at the opposite position. Next, the individual skysubtracted cubes were combined, centroiding on the primary. The individual wavelength planes were shifted and aligned on the primary such that the final path of the companion through the data cube was a straight trajectory. Due to the small angular separation between the primary and secondary, the halo of the primary was fit with a radial profile and subtracted from each wavelength plane. Images at $J, H$, and $K$, collapsed in wavelength over the entire band, before and after the subtraction process are shown in Fig. 1. The measured FWHM of the target was stable at $\sim 3.5$ pixels. Following the radial subtraction, the companion spectrum was constructed by extracting the flux in an aperture of 4.5 pixel radius that encompasses the majority of the flux in a consistent manner. Given the stability of the primary FWHM throughout each wavelength band, variations of the Strehl ratio across a band seem minimal and should not systematically bias the extracted spectrum.

Correction for telluric absorption and the detector response was accomplished by dividing the observed target spectra by those of early-type standard stars with interpolation across intrinsic features. The shape of the standard was then removed by multiplication by a black-body at the appropriate effective temperature (see Table 1 ). The extracted $J$ and $H K$ spectra of $2 \mathrm{M} 1207 \mathrm{~b}$ were flux calibrated using the $J$ and $K_{\mathrm{S}}$-band 


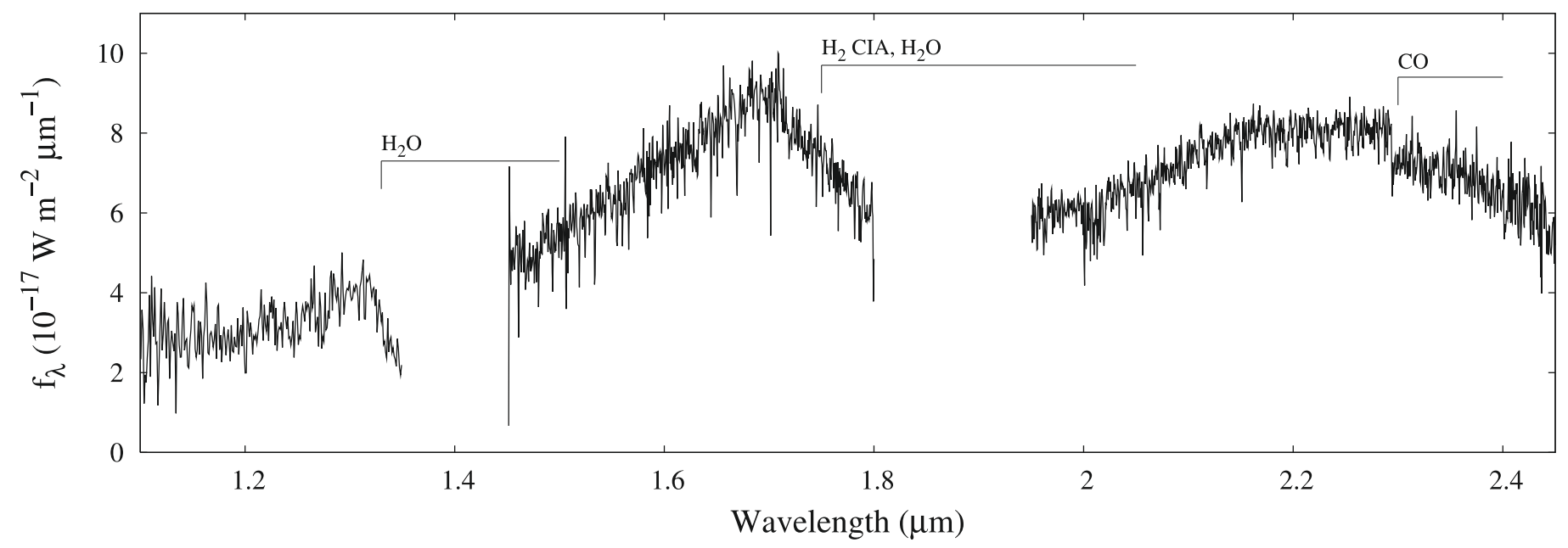

Fig. 2. The spectrum of $2 \mathrm{M} 1207 \mathrm{~b}$ covering the $J, H$, and $K$ passbands flux calibrated using the apparent magnitudes of Chauvin et al. (2004) and Mohanty et al. (2007). Key features are indicated.

photometry of Mohanty et al. (2007) and Chauvin et al. (2004), respectively. The expected ratio of detected photons was calculated by convolving the observed spectrum and that of Vega (Mountain et al. 1985; Hayes 1985) with the 2MASS response curves (Carpenter 2001), assuming Vega to have zero magnitude in both filters.

The flux calibrated near-IR spectrum of 2M1207 b is shown in Fig. 2. Over the $J$-band the signal-to-noise ratio is $\sim 7$ per resolution element, increasing to $\sim 13$ across the $H$-band and $\sim 24$ over the $K$-band. The depression of $J$ due to atmospheric dust absorption, the two prominent water absorption bands between the near-IR passbands, and the $\mathrm{CO}$ absorption at the red end of the $K$-band are clearly evident.

To estimate the effective temperature and surface gravity of $2 \mathrm{M} 1207 \mathrm{~b}$, we compared the flux calibrated $J H K$ spectrum to the AMES-Dusty model grid (Allard et al. 2001) with $T_{\text {eff }}=500-4000 \mathrm{~K}$ and $\log g=3.5-6.0$. The model spectra were smoothed to the same resolution as the observations and interpolated to the same dispersion. Using the same least-squares statistic as Mohanty et al. (2007) and allowing the model flux to be scaled to find the best global fit, we identified the best fitting models. Calibrating the model spectra to the flux as would be observed from Earth requires a scale factor which depends on the radius of the object and its distance. Since we have flux calibrated the observed spectrum, we can extract an estimate of the radius implied for the closest match between each model and the observations.

\section{Results and discussion}

The larger coverage in wavelength and higher signal-to-noise enables an investigation of the atmosphere and origin of this young, planetary mass companion. The flux calibrated spectrum of $2 \mathrm{M} 1207 \mathrm{~b}$ is compared with previous observations of field and young low mass objects and with theoretical model atmospheres. The overall shape is significantly different to field L dwarfs, with a marked depression in the $J$-band as shown in Fig. 3. The $H$-band continuum exhibits a distinct triangular shape unlike the older, higher surface gravity objects, and the $K$-band peaks at $\sim 2.3 \mu \mathrm{m}$ (as suggested by Mohanty et al. 2007) rather than at $\sim 2.15 \mu \mathrm{m}$ as with field L dwarfs, and the region associated with the peak is more clearly defined in these higher resolution data.

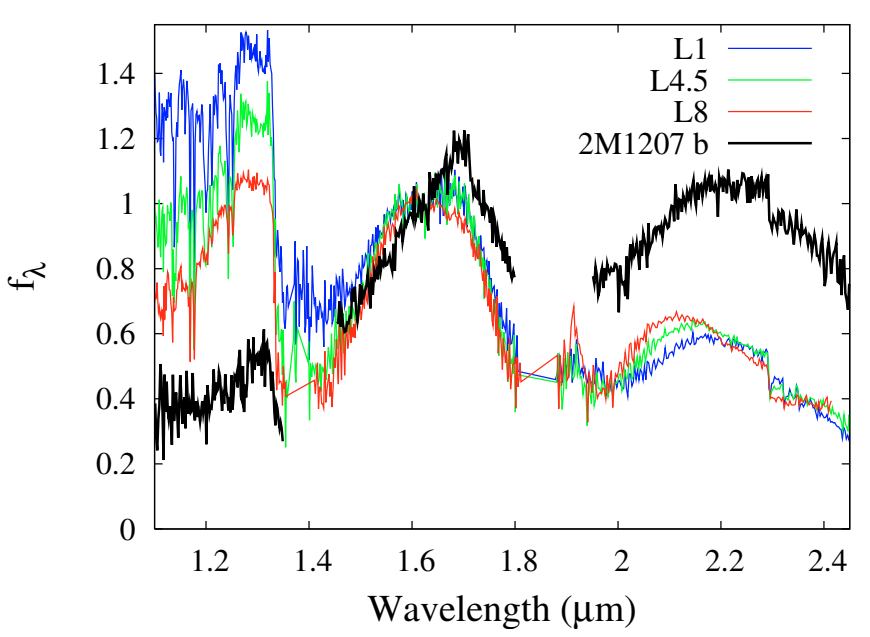

Fig. 3. The $J, H, K$ spectrum of $2 \mathrm{M} 1207 \mathrm{~b}$ (black line) and several comparison spectra of field L dwarfs with spectral types L1 (blue), L4.5 (green), and L8 (red). All spectra are normalised over the 1.60-1.65 $\mu \mathrm{m}$ range.

The spectrum of 2M1207 b is unlike field L-dwarfs spanning a range of spectral types, but the young age of the TW Hydra association (8 Myr) complicates the comparison. In Fig. 4, the spectrum of $2 \mathrm{M} 1207 \mathrm{~b}$ is plotted with several spectra of low mass members of Upper Sco (Lodieu et al. 2008), with spectral types ranging from $\mathrm{M} 8-\mathrm{L} 2$, and with the borderline brown dwarf/planet companion AB Pic b (Bonnefoy et al. 2010). Even amongst young, low-mass objects in the 5 Myr Upper Sco region, the 2M1207 b spectrum appears distinct in the shape and relative fluxes of the continuum across the different bands. No Upper Sco member has a lower $J$-band relative to $H$-band and the triangular shape of the $H$-band is only beginning to develop by the L2 spectral type. The spectral sequence shown in Fig. 4 suggests that $2 \mathrm{M} 1207 \mathrm{~b}$ is one of the coolest young companions imaged. The most analogous object to $2 \mathrm{M} 1207 \mathrm{~b}$ is the companion $\mathrm{AB}$ Pic $\mathrm{b}$ which also shows some of the key features present in $2 \mathrm{M} 1207 \mathrm{~b}$. Since the $J$-band data required binning, the spectral resolution is not yet high enough to identify and measure 


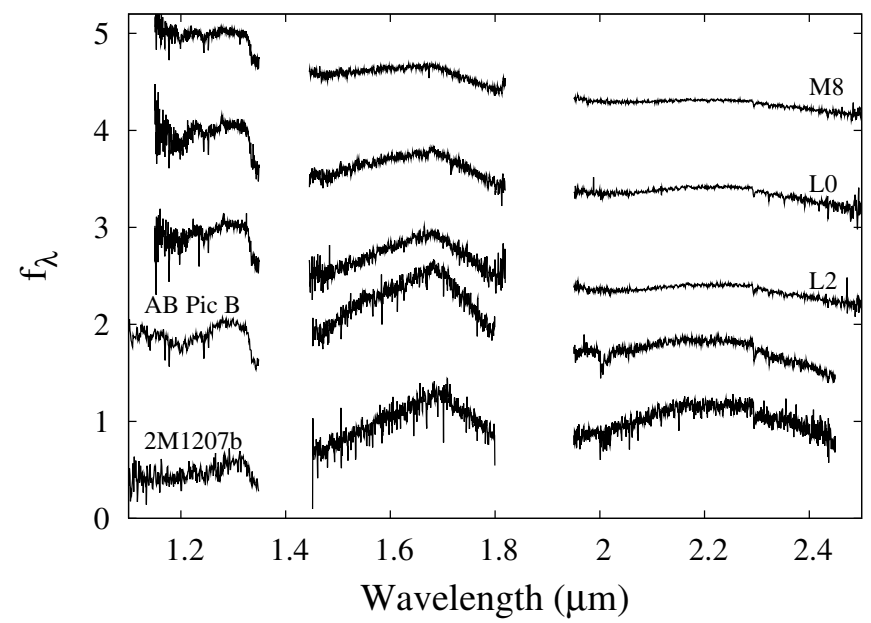

Fig. 4. The $J, H, K$ spectrum of $2 \mathrm{M} 1207 \mathrm{~b}$ (bottom) and several comparison spectra of young (5-30 Myr) objects in Upper Sco of spectral types (from the top): M 8, L0, L2, and L0-L1 (AB Pic b). The spectra have been flux calibrated as in Fig. 3, and then offset by an integer for clarity.

absorption lines, though future observations with more signalto-noise could investigate the depths of the Na I and K I lines.

A series of spectral indices based on ratios of the flux of different regions of the continuum have been developed to characterise the spectral type of cool objects (McLean et al. 2003; Slesnick et al. 2004; Allers et al. 2007). We measured these for $2 \mathrm{M} 1207 \mathrm{~b}$ and summarise the results in Table 2 for the Allers et al. (2007) $\mathrm{H}_{2} \mathrm{O}$ and gravity sensitive indices, the Slesnick et al. (2004) $\mathrm{H}_{2} \mathrm{O}$ and $\mathrm{FeH}$ indices, and the McLean et al. (2003) $\mathrm{H}_{2} \mathrm{O}$ indices. These suggest a spectral type in the broad range M 8.5-L4, while the gravity sensitive index is consistent with that found for other young brown dwarfs. The discrepancy in inferred spectral types is not surprising given the unusual overall shape of the spectrum and highlights the importance of obtaining larger wavelength coverage of flux calibrated spectra in assigning spectral types and their uncertainties.

The flux calibrated 2M1207 b spectrum with $R \sim 300$ at $J$ and $R \sim 1500$ at $H K$ is shown in Fig. 5 along with the best fit to the model DUSTY spectrum (Allard et al. 2001). Available models with temperatures higher and lower by $100 \mathrm{~K}$ are also plotted in Fig. 5. The best fit, estimated by a least-squares fit to the grid of DUSTY models, and confirmed by visual examination is shown in the middle panel of Fig. 5 with $T_{\text {eff }}=1600 \mathrm{~K}$ and $\log g=4.5$. The uncertainty in temperature is clearly less than $\pm 100 \mathrm{~K}$, but the ability to discriminate surface gravity variations with the broad morphology and flux level of the spectrum is less than for effective temperature. Consequently, $\log g=4.5$ is only marginally favoured over $\log g=3.5$ or 5.5 due to the uncertainty on the flux level of the $J$-band spectrum resulting from the photometry uncertainty of $0.2^{\mathrm{m}}$. While the location of the peak in the $H$-band is consistent with the models, the observed slopes on either side are not as steep as the model predicts, possibly due to difficulties modelling the $\mathrm{H}_{2}$ collisionally-induced absorption. If the precision of the $J$-band photometry could be improved, the model variations would allow surface gravity to be constrained to $\sim 0.5 \mathrm{dex}$, important to test one formation model. These physical parameters are consistent with those measured previously (Mohanty et al. 2007), though the inclusion of the $J$-band and the higher signal-to-noise of these data makes the fitting more secure.
Table 2. Spectral indices.

\begin{tabular}{llllll}
\hline \hline Index & $\mathrm{H}_{2} \mathrm{O}$ & grav. & $\mathrm{H}_{2} \mathrm{O}-1$ & $\mathrm{H}_{2} \mathrm{O}-2$ & $\mathrm{FeH}$ \\
Value & 1.17 & 1.01 & 0.660 & 0.831 & 0.877 \\
SpT & L0 & low grav. & L0 & L1.5 & $\mathrm{M} 8.5$ \\
Index & $\mathrm{H}_{2} \mathrm{O}-A$ & $\mathrm{H}_{2} \mathrm{O}-\mathrm{B}$ & $\mathrm{H}_{2} \mathrm{O}-\mathrm{C}$ & $\mathrm{H}_{2} \mathrm{O}-\mathrm{D}$ & \\
Value & 0.593 & 0.724 & 0.733 & 0.817 & \\
SpT & L2.5 & L3 & L0 & L4 & \\
\hline
\end{tabular}

References. From Allers et al. (2007); Slesnick et al. (2004); and McLean et al. (2003).

We did not compare the data to the COND models since they do not adequately reproduce the previous lower resolution $H$ - and $K$-band spectrum (Mohanty et al. 2007). In addition to the treatment of dust condensation and settling, the inclusion of non-equilibrium $\mathrm{CO} / \mathrm{CH}_{4}$ chemistry can significantly alter the shape of the continuum emission (Fortney et al. 2008), particularly in the $K$ - and $L$-bands. A suitable parameterisation of the eddy diffusion coefficient for such models may reproduce the shape of the observed spectrum, however, the flux level for the $T_{\text {eff }}=1400 \mathrm{~K}$ (the hottest shown), $K_{z z}=4$ non-equilibrium atmosphere shown in Fortney et al. (2008) is still significantly higher than $2 \mathrm{M} 1207 \mathrm{~b}$; for the range of $K_{z z}, \log g$, and metallicity presented for the $T_{\text {eff }}=1400 \mathrm{~K}$ case, the $K$-band magnitude varies by up to $0.7 \mathrm{mag}$.

As noted previously, to match the predicted spectral model fluxes to the observed photometry, either 2M1207 b is underluminous by a factor of $\sim 10$ with a radius of $\sim 0.16 R_{\odot}$, or has a smaller than expected radius. The required radius to explain the photometry is $\sim 0.052 R_{\odot}$, about one third of the radius of $\sim 0.16 R_{\odot}$ from the DUSTY evolutionary model prediction for an object of $T_{\text {eff }}=1600 \mathrm{~K}$ at $5-10 \mathrm{Myr}$, and significantly smaller than any predicted radius for a partially degenerate object above $1 M_{\text {Jup }}$ (Chabrier et al. 2009).

The origin of 2M1207 b and its underluminous flux is uncertain, and different scenarios have been proposed for the specific case of 2M1207 b: an edge-on disk (Mohanty et al. 2007) and the result of a collision of protoplanets (Mamajek \& Meyer 2007). More generally, the impact on the luminosity due to initial conditions and the early accretion history of young objects with ages comparable to 2M1207 b, has been investigated (Marley et al. 2007; Baraffe et al. 2009). The protoplanet collision hypothesis predicts that the object is much smaller and should have a surface gravity of 3.0 rather than 4.5. The upper panel of Fig. 5 shows the data with DUSTY spectral models with three different effective temperatures for a lower surface gravity of $\log g=3.5$ (since $\log g=3.0$ is not available). The data favour the higher surface gravity $\log g=4.5$ value, though increasing the $J$-band flux by one sigma would make the data consistent with the $\log g=3.5$ model. A $J$-band magnitude difference with smaller uncertainties would be required to entirely rule out the collision scenario.

For an initial investigation into the edge-on disk possibility, we compared the photometry and spectra with synthetic spectra generated by the TORUS and MCFOST codes (Pinte et al. 2009). A central object simulated by the $T_{\text {eff }}=1600 \mathrm{~K}$ DUSTY atmosphere was encircled with a disk $10 \mathrm{AU}$ in radius, consistent with the physical separation of the 2M1207 pair. The disk was constructed with canonical values for flaring, surface density power law, dust size distribution and chemistry. The inclination was allowed to vary from edge-on to the $\sim 60$ degree limit suggested for the geometry of the primary disk. Within 


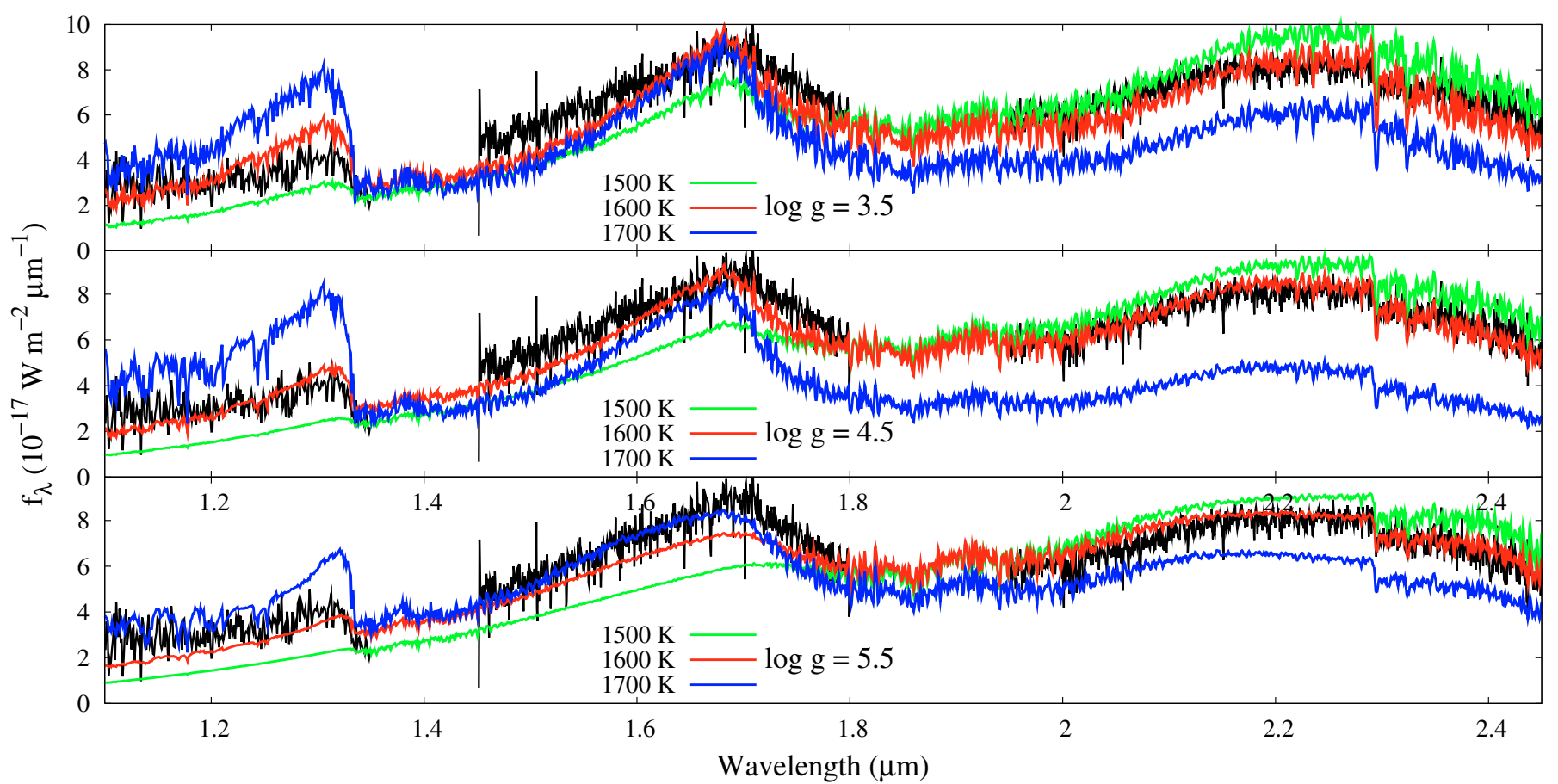

Fig. 5. The spectrum of $2 \mathrm{M} 1207 \mathrm{~b}$ covering the $J, H$, and $K$ passbands (black lines). Middle panel: the best-fit DUSTY model with $T_{\text {eff }}=1600 \mathrm{~K}$ and $\log g=4.5$ in red along with the $1500 \mathrm{~K}$ (green line) and $1700 \mathrm{~K}$ (blue line) $\log g=4.5$ models. Top and bottom panels: DUSTY models of the same effective temperature as the middle panel, but with surface gravity of $\log g=3.5$ and $\log g=5.5$, respectively.The observed spectrum has been flux calibrated using the apparent magnitudes of Chauvin et al. (2004) and Mohanty et al. (2007). While the DUSTY models reproduce the general morphology, the flux scaling necessary implies a radius of $\sim 0.052 R_{\odot}$ at a distance of $53 \mathrm{pc}$, significantly smaller than any predicted radius of an ultracool dwarf. Discussion of this unphysical radius, or equivalently low luminosity is given in Sect. 5.

the limited range of disk parameters searched, scattering alone could not explain the flux level of the central object since the predicted scattered flux was only a few per-cent, rather than the required $\sim 10 \%$. For comparison, the T Tauri stars HH 30 and HK Tau with imaged edge-on disks (Stapelfeldt et al. 1998) are fainter by 3-4 mag relative to the photospheric levels, consistent with a scattered flux level of a few per-cent or less. Thus, among disk scenarios, grey extinction from a disk composed of larger grains than considered in the simulations is favoured over scattering off the surface of an optically thick disk; grain sizes larger than $4 \mu \mathrm{m}$ have been suggested as a possibility (Mohanty et al. 2007). Additionally, extinction of a hotter object is not able to explain the wavelength dependence of the photometry over the entire $0.9-3.6 \mu \mathrm{m}$ range.

In summary, the SINFONI spectrum of 2M1207 b represents the highest resolution, largest wavelength coverage spectrum of a young, planetary mass companion. The overall shape is distinct from field and young cluster L-dwarfs, with substantially different ratios of flux across the bands, a triangular shape to the $H$-band continuum, and a peak at longer wavelengths in the $K$-band. Spectral indices developed for cool objects give conflicting values, indicating the unusual nature of this object. The spectrum is best fit by a DUSTY model atmosphere with $T_{\text {eff }}=1600 \mathrm{~K}$ and $\log g=4.5$, though it is not yet possible to exclude the $\log g=3.0$ atmosphere predicted by one formation model. We find that the apparent underluminosity of 2M1207 b is consistent with extinction from large dust grains in a nearly edge-on disk, but more precise $J$-band and longer wavelength photometry and spectroscopy will help to determine the nature of this important object. This spectrum of 2M1207 b will serve as a key comparison for upcoming near-infrared spectroscopy of planets imaged around stellar hosts.

Acknowledgements. We gratefully acknowledge funding for a Leverhulme research project grant to J.P. (ID20090041) and a studenship for R. J. DR. (ST/F 007124/1) from the Science and Technology Facilities Council (STFC). We thank T. Harries, C. Pinte, and N. Mayne for providing synthetic SEDs for comparison with the data. We thank J. Fortney, R. White and I. Baraffe for scientific discussions and the referee for helpful comments.

\section{References}

Allard, F., Hauschildt, P. H., Alexander, D. R., Tamanai, A., \& Schweitzer, A. 2001, ApJ, 556, 357

Allers, K. N., Jaffe, D. T., Luhman, K. L., et al. 2007, ApJ, 657, 511

Baraffe, I., Chabrier, G., Allard, F., \& Hauschildt, P. H. 2002, A\&A, 382, 563

Baraffe, I., Chabrier, G., Barman, T. S., Allard, F., \& Hauschildt, P. H. 2003, A\&A, 402, 701

Baraffe, I., Chabrier, G., \& Gallardo, J. 2009, ApJ, 702, L27

Bonnefoy, M., Chauvin, G., Rojo, P., et al. 2010, A\&A, 512, A52

Bonnet, H., Abuter, R., Baker, A., et al. 2004, The Messenger, 117, 17

Boss, A. P. 1997, Science, 276, 1836

Carpenter, J. M. 2001, AJ, 121, 2851

Chabrier, G., Baraffe, I., Allard, F., \& Hauschildt, P. 2000, ApJ, 542, 464

Chabrier, G., Baraffe, I., Leconte, J., Gallardo, J., \& Barman, T. 2009, in AIP Conf. Ser. 1094, ed. E. Stempels, 102

Charbonneau, D., Brown, T. M., Noyes, R. W., \& Gilliland, R. L. 2002, ApJ, 568,377

Charbonneau, D., Allen, L. E., Megeath, S. T., et al. 2005, ApJ, 626, 523

Chauvin, G., Lagrange, A., Dumas, C., et al. 2004, A\&A, 425, L29

Chauvin, G., Lagrange, A., Zuckerman, B., et al. 2005, A\&A, 438, L29

Deming, D., Seager, S., Richardson, L. J., \& Harrington, J. 2005, Nature, 434, 740

Ducourant, C., Teixeira, R., Chauvin, G., et al. 2008, A\&A, 477, L1

Dumas, C., Kaufer, A., \& Hainaut, O. 2007, in SINFONI data reduction cookbook, VLT-MAN-ESO-14700-4037 
Eisenhauer, F., Abuter, R., Bickert, K., et al. 2003, in SPIE Conf. Ser. 4841, 1548 Fortney, J. J., Marley, M. S., Saumon, D., \& Lodders, K. 2008, ApJ, 683, 1104 Gebbinck, M. K., Ballester, P., \& Peron, M. 2007, in VLT Gasgano User's Manual, VLT-PRO-ESO-19000-1932

Gizis, J. E. 2002, ApJ, 575, 484

Grillmair, C. J., Burrows, A., Charbonneau, D., et al. 2008, Nature, 456, 767

Hayes, D. S. 1985, in Calibration of Fundamental Stellar Quantities, ed. D. S. Hayes, L. E. Pasinetti, \& A. G. D. Philip, IAU Symp., 111, 225

Janson, M., Bergfors, C., Goto, M., Brandner, W., \& Lafrenière, D. 2010, ApJ, 710, L35

Kalas, P., Graham, J. R., Chiang, E., et al. 2008, Science, 322, 1345

Lodieu, N., Hambly, N. C., Jameson, R. F., \& Hodgkin, S. T. 2008, MNRAS, 383,1385

Mamajek, E. E., \& Meyer, M. R. 2007, ApJ, 668, L175

Marley, M. S., Fortney, J. J., Hubickyj, O., Bodenheimer, P., \& Lissauer, J. J. 2007, ApJ, 655, 541

Marois, C., Macintosh, B., Barman, T., et al. 2008, Science, 322, 1348

McLean, I. S., McGovern, M. R., Burgasser, A. J., et al. 2003, ApJ, 596, 561

Modigliani, A., Mirny, K., Ballester, P., \& Peron, M. 2009, in SINFONI Pipeline User Manual, VLT-MAN-ESO-19500-3600
Mohanty, S., Jayawardhana, R., Huélamo, N., \& Mamajek, E. 2007, ApJ, 657, 1064

Mountain, C. M., Selby, M. J., Leggett, S. K., Blackwell, D. E., \& Petford, A. D 1985, A\&A, 151, 399

Pinte, C., Harries, T. J., Min, M., et al. 2009, A\&A, 498, 967

Pollack, J. B., Hubickyj, O., Bodenheimer, P., et al. 1996, Icarus, 124, 62

Pont, F., Knutson, H., Gilliland, R. L., Moutou, C., \& Charbonneau, D. 2008, MNRAS, 385, 109

Richardson, L. J., Deming, D., Horning, K., Seager, S., \& Harrington, J. 2007, Nature, 445, 892

Sing, D. K., Vidal-Madjar, A., Lecavelier des Etangs, A., et al. 2008, ApJ, 686, 667

Slesnick, C. L., Hillenbrand, L. A., \& Carpenter, J. M. 2004, ApJ, 610, 1045

Song, I., Schneider, G., Zuckerman, B., et al. 2006, ApJ, 652, 724

Stapelfeldt, K. R., Krist, J. E., Menard, F., et al. 1998, ApJ, 502, L65

Swain, M. R., Vasisht, G., \& Tinetti, G. 2008, Nature, 452, 329

Thatte, N. A., Tecza, M., Eisenhauer, F., et al. 1998, in SPIE Conf. Ser. 3353, ed. D. Bonaccini, \& R. K. Tyson, 704 